\title{
Assessment and testing of seismic hazard for planned mining sequences
}

DA Malovichko Institute of Mine Seismology, Australia

\begin{abstract}
An approach to assess seismic and ground motion hazard associated with scenario(s) of future mining is suggested. The key element of the approach is the modelling of expected seismicity using the Salamon-Linkov method (Malovichko \& Basson 2014) and combining the catalogues of modelled and observed seismic events. The modelling domain is discretised and it is assumed that the potency frequency distribution in each grid point can be described by the Upper Truncated model. The upper cutoff potency $P_{\max }$ and slope $\beta$ are presumed the same for all grid points. $P_{\max }$ is estimated from the combined (observed and modelled) seismic catalogue using record theory (Section 3.4 of Mendecki 2016) and the slope $\beta$ is evaluated from observed data. The parameter $\alpha$ of the Upper Truncated model is inferred from cumulative potency, which is calculated for each grid point using the combined catalogue of events. The probabilities of occurrence of events exceeding a specific potency are derived for each grid point, assuming that the temporal occurrence of events follows a Poisson distribution. The assessment of ground motion hazard is based on Monte Carlo simulation of ground motion accounting for uncertainties in the Ground Motion Prediction Equation and variation of expected seismicity according to the described above estimate of seismic hazard.

There are two utilities of assessment of seismic and ground motion hazard in mines. Firstly, the calculated probabilities can be categorised in terms of the hazard likelihoods specified in the risk assessment matrix established at the mine. This can guide a geotechnical engineer in the required actions. Secondly, the evaluated seismic and ground motion hazard can be rigorously tested after the period of forecast is expired and the actual seismic response to planned mining is recorded. The testing procedures established in crustal seismology can be adopted. The poor performance of the forecast needs to be explained in geomechanical terms and corresponding settings of the modelling of seismicity have to be updated.
\end{abstract}

The suggested approach of forecasting the seismic and ground motion hazard, as well as retrospective testing of the seismic hazard, are illustrated using planned mining sequence and seismic data from Renison mine, Australia.

Keywords: seismicity, modelling, seismic hazard, ground motion hazard

\section{Introduction}

Seismic hazard is defined as a probability that a potentially damaging event will occur in a given volume within a given period of time in future (Mendecki 2016). In crustal seismology, the seismic hazard is usually assessed under the assumption that characteristics of future earthquakes (location, size, mechanism) will be similar to the ones recorded in the past. This is legitimate as the earthquakes are driven by tectonic forces, which show minor changes over the time of instrumentally recorded data. This is not the case in mines, where seismicity is driven by redistribution of stresses by excavations and the future mining plays an essential role in the characteristics of large seismic events that may occur. Thus, the probabilities of potentially damaging events inferred from recorded seismic data can only be used to characterise the current hazard state (Wesseloo 2013).

The effect of future mining on seismicity can be quantified using numerical modelling of stresses and strains. The cell evaluation method was suggested by Beck \& Brady (2002), where the frequency of recorded seismic events in the cells of a regular grid is correlated with modelling parameters (for instance, 
Modelled Ground Work) calculated for historical mining steps. The established correlation makes it possible to assess the probabilities of seismic events for the models of future mining. Later papers recommend using other modelling parameters - Dissipated Plastic Energy (Beck et al. 2007; Arndt et al. 2013) and the Rate of Energy Release (Levkovitch et al. 2013).

Methods of explicit modelling of seismicity associated with mining have been discussed in a number of publications (Salamon 1993; Board 1994; Spottiswoode 2001; Sellers \& Napier 2001; Linkov 2005, 2013; Spottiswoode \& Drummond 2014). These methods also provide a way to forecast the expected seismic response for the planned mining sequence(s) and therefore evaluate seismic hazard.

This paper describes the approach to assess seismic hazard based on explicit modelling of expected seismicity using the Salamon-Linkov method (Malovichko \& Basson 2014). It also presents how the assessed seismic hazard can be translated to ground motion hazard, which is the probability that strong ground motion associated with a seismic event will exceed a given threshold in a given place within a given period of time in future (Mendecki 2016).

The seismic hazard forecast for a particular mining sequence can be tested against the recorded data after the sequence is completed. A number of testing procedures developed in crustal seismology can be utilised for this. If several alternative forecasts of hazard were considered (e.g. obtained using various parameters of modelling of seismicity) then the better ones can be identified, which effectively represents the calibration of modelling parameters. Discussion of the testing of forecasts of seismic hazard constitutes the final part of the paper.

\section{Assessment of hazard}

Data from Renison mine, Australia is used to illustrate the proposed approach of assessment of seismic and ground motion hazard. This mine exploits narrow veins striking from north to south and steeply dipping to the east. The in situ maximum principal stress is sub-horizontal and oriented approximately parallel to the veins.

The seismic system operating at the mine has a 3D configuration of triaxial geophones installed in boreholes. The recorded seismic data is of high quality which allows for the routine evaluation of not only basic source parameters of events (location, origin time, seismic potency and seismic energy), but also source mechanisms.

The sequence of quarterly mining steps shown in Figure 1 was planned at this mine in the beginning of 2016.

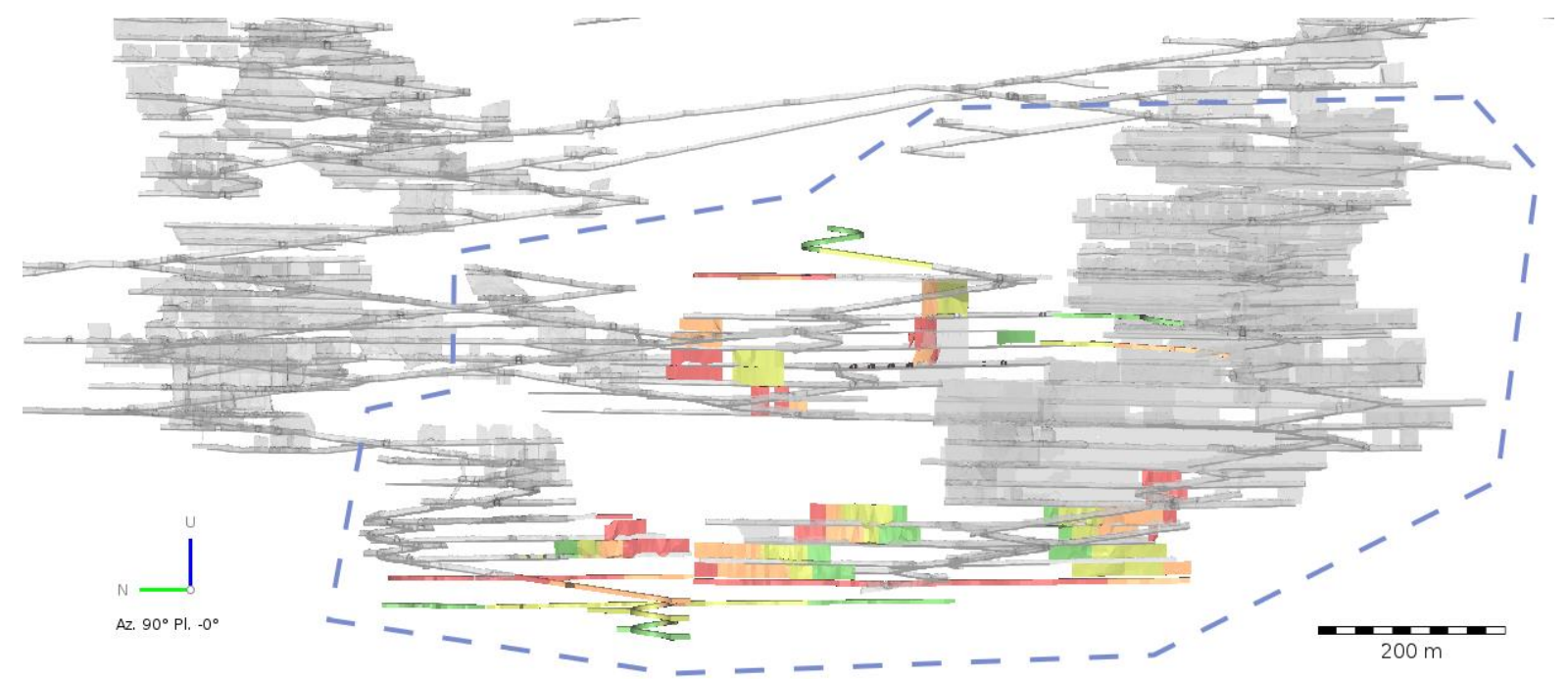

Figure 1 Existing mining (grey wireframes) and four planned quarterly mining steps (red - January to March 2016, green - October to December 2016). The blue dash polygon outlines the region where the hazard was assessed 


\subsection{Modelling of expected seismicity}

\subsubsection{Method}

The method to model seismicity associated with mining was suggested in the pioneering work of Salamon (1993). The ideas of Salamon were extensively elaborated on and improved by Linkov $(2005,2013)$. His computational framework allows one to model both seismic and aseismic events, and to reproduce time effects of the induced and triggered seismicity (e.g. aftershock sequences).

The main idea of the methods of Salamon and Linkov is to identify possible failure of flaws in the elastic rock mass model and then to transfer the parameters of modelled failure into a seismological domain. As mentioned in Linkov (2013), the method may utilise an arbitrary numerical code that solves an elastostatic problem (Boundary Element Method, Finite Element Method and the like). The original method of Salamon was 'passive', i.e. the pre-calculated total (in situ + mining-induced) stress is used to check the fulfilment of failure criterion for the flaws pre-seeded in the model. In Linkov's version each flaw plays an active role in the redistribution of the stress field.

A variation of Linkov's approach, called the Salamon-Linkov method, was suggested by Malovichko and Basson (2014). It has the following differences with the original method:

- The method does not require a priori assumptions about sizes, orientations and locations of flaws, thus reducing the amount of input data. The method employs the testing of various orientations and directions of flaws and the identification of the ones that are favourable for slip according to prescribed failure criteria. The spatial heterogeneity and anisotropy of rock mass strength is accounted for by the appropriate setting of failure criteria, e.g. specific failure criterion can be used for the flaws with specific locations and orientations to describe faults or joint sets.

- The flaws are discretised into smaller subflaws. This accounts for the complexity of the shapes of the flaws and provides more accurate modelling of their effect on the stress field.

- Only shear flaws are considered. The original formulations of the methods of Salamon and Linkov adopted tensile flaws and these were interpreted as events with explosive source mechanisms. This conflicts with the observations, where spalling events generally have the opposite (implosive) mechanisms. The accompanying convergence of nearby excavations needs to be taken into account when deriving source sizes and mechanisms for tensile flaws. This is not implemented in the current version of the Salamon-Linkov method and will be addressed in its future development.

The discussion of differences between the methods can also be found in Linkov et al. (2016).

Our current implementation of the Salamon-Linkov method is based on the Indirect Boundary Element Method (IMS in-house code). The Fictitious Forces (FF) models of excavations were used in the illustrated case. The flaws are represented by Displacement Discontinuity (DD) elements. These make the model inelastic.

\subsubsection{Calibration}

As input information, the Salamon-Linkov method requires parameters of in situ stress and failure criteria. The latter can be non-uniform and anisotropic (e.g. particular orientations or 3D surfaces may have failure criteria, different from the failure criterion of the rock mass). It is possible to model expected seismicity for future mining using input parameters suggested by the mine. However in general it is highly preferable to start with the testing and adjustment (as necessary) of the provided parameters by modelling seismicity for historical mining steps and comparing it with the corresponding actual recorded seismicity. The duration of historical steps in such a calibration exercise has to be chosen similar to the duration of future mining steps.

The comparison of modelled and observed seismicity can be done in terms of location, sizes and mechanisms of events. In the process of tuning the model, usually the location and source mechanisms are sensitive to the parameters of in situ stress and failure criteria. The sizes of events are mostly sensitive to failure criteria. 
During the calibration it is better to start with the simplest possible model and then try to reduce the differences between the modelled and observed seismicity either by changing the orientation of in situ principal stresses or by modifying of failure criteria (including adding of geological structures in the model). For example, in the case of Renison mine the calibration was done in three iterations:

- Iteration 1) Initially, uniform failure criteria recommended by the mine was used for the entire rock mass. The modelled seismicity clustered too close to the stopes compared to the observed events. The sizes of the modelled events (in terms of seismic potency) were also smaller than the observed ones. The mechanisms of the observed events show clustering of nodal planes around a specific orientation correlated with one of the mapped structures (Footwall North set).

- Iteration 2) A low strength orientation (Footwall North joint set) was included in the model and provided better match between the observed and modelled seismicity in terms of sizes, location and mechanisms of events in the footwall and around the orebody. However the model did not simulate a deficit of potency in the observed seismicity in the immediate hanging wall, nor the observed small-size seismicity spread into the hanging wall quite far from the orebody along a sub-planar zone.

- Iteration 3) A low strength perforated creep surface in the hanging wall of the orebody was included in the model. This allowed the simulation of the deficit of observed potency in the hanging wall and the small-size remote events along the sub-planar zone.

A number of calibration exercises have been done at different mines in which the simulated seismicity was derived under the assumptions that all episodes of failure in the model (DD elements in the BEM) occur seismically. In all of these, the modelled seismicity demonstrated the scaling of sizes of the events (e.g. left plot of Figure 2), although it generally did not exactly replicate the size distribution of observed seismicity. Calibration allows the matching of the sizes of the largest modelled events with the largest recorded events, however in most cases the modelling delivered more medium-size events than those recorded in reality. This makes sense taking into account that (a) there is a limitation on the smallest size of modelled events imposed by the smallest DD in the model, and (b) all failures in the models are considered to happen seismically. As it is shown by Linkov (2013), larger cracks have a lower shear rigidity compared to smaller cracks. Provided that the shear softening modulus is the same for large and small cracks, the former have a greater chance to fail in an unstable manner (seismically) compared to the later. Conversely, the smaller cracks are more prone to stable failure (aseismic events). Therefore, it will be legitimate to correct for excessive modelled potency in cases of small-size events. This can be done by comparing Upper Truncated models of potency frequency distribution of observed events:

$$
N_{(o)}(\geqslant P)=\alpha_{(o)}\left(P^{-\beta_{(o)}}-P_{\max }^{-\beta_{(o)}}\right)
$$

with the similar distribution of modelled events:

where:

$$
N_{(m)}(\geqslant \tilde{P})=\alpha_{(m)}\left(\tilde{P}^{-\beta_{(m)}}-P_{\max }^{-\beta_{(m)}}\right)
$$

$N(\geqslant P)$ is the number of events with seismic potency $\geqslant P$.

subscripts $(o)$ and $(m)$ denote observed and modelled data, respectively.

$\alpha, \beta$ and $P_{\max }$ are the parameters of the Upper Truncated model.

One way to correct the modelling data is to decimate the catalogue of simulated events. However, it will introduce uncertainty into the modelling results - some medium-size events can disappear from the catalogue or remain in it depending on the implementation of the decimation procedure. This may complicate the spatial analysis of the data discussed further. An alternative correction is to apply the following transformation to the modelled potencies $\tilde{P}$ :

$$
P=\left[\alpha_{(o)} / \alpha_{(m)}\right]^{1 / \beta_{(o)}} \tilde{P}^{\beta_{(m)} / \beta_{(o)}}
$$


The results of such a transformation are illustrated in Figure 2 for the modelled seismicity shown in Figure 3. This transformation is the preferred approach for now.

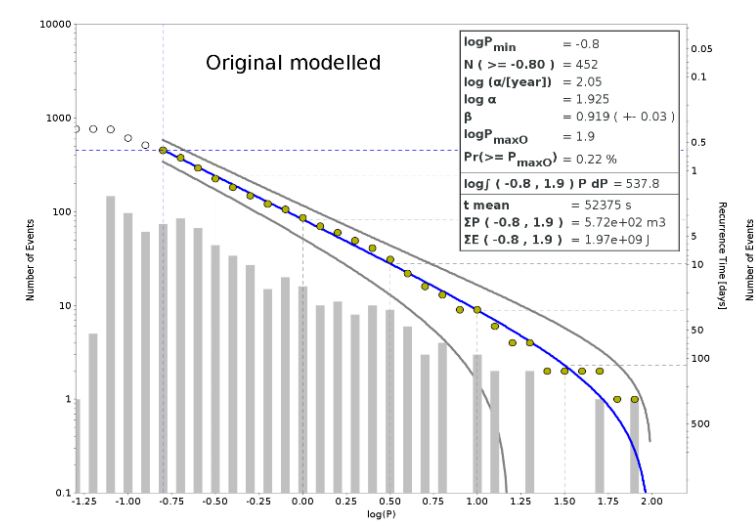

(a)

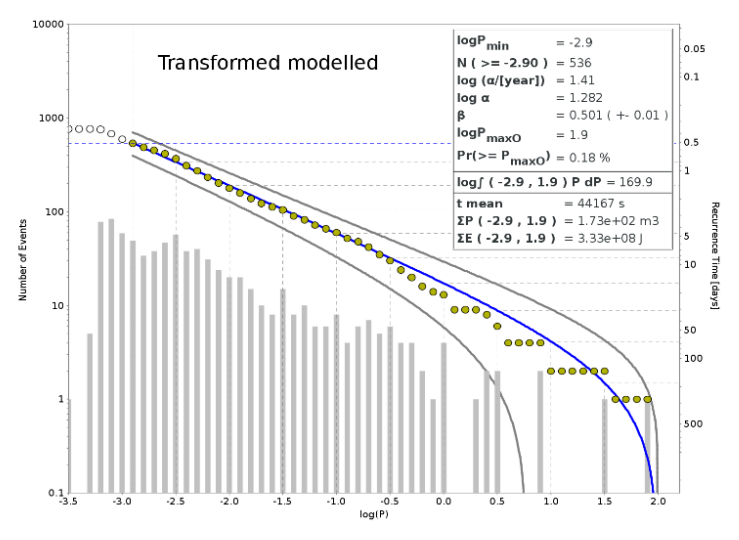

(b)

Figure 2 Correction of sizes of modelled seismic events. Distribution of $\log P$ for the original modelled dataset (a); and for the same dataset with corrected potencies (b)

\subsubsection{Application}

The results of the application of Salamon-Linkov method to the planned mining steps shown in Figure 1 are presented in Figure 3. The modelled events are assigned to the middle of mining steps - 15 February 2016, 15 May 2016, etc. The sizes (potencies) of events are corrected according to procedure described in the previous subsection.

In general, the modelled seismicity is clustered around the planned stopes, however some events appear far from them (for instance, events in the shallower southern part of the mine). These are associated with the extensive failure zones in the footwall and hanging wall of the large mined-out area. The fracturing on the perimeter of this zone is not only induced by nearby stoping, but it is also triggered by more distant mining.

Another remarkable feature is the variation of the scattering and sizes of expected seismic events in different parts of the mine. The planned mining in the northern part of the mine occurs in more solid ground, which results in the weaker localised expected seismic response. The planned mining in the deeper southern part of the mine extends the large mined-out stoping area above. Thus, the associated seismicity is expected to be scattered further from the orebody and include larger events.

\subsection{Quantification of seismic hazard}

The modelled seismic events cannot be interpreted as the only possible outcome of the considered mining scenario. To get a probabilistic description of the expected seismicity it would be necessary to repeat the simulation multiple times for plausible variations of input and calculation parameters (in situ stress, failure criteria, weak structures, etc.). However, this approach is time-consuming and not feasible with current computational power.

Therefore, the seismic hazard is here derived from a single available sample of expected seismicity (i.e. seismicity shown in Figure 3). The following procedure is suggested for this:

- The catalogue of modelled seismic events is combined with the catalogue of observed events. The content of the combined catalogue for the demonstration example is shown in Figure 4. The spatial and temporal span of the catalogue of observed events needs to be selected considering the mechanics of large events in the past and their relevance to the expected seismicity in future. For instance, in the case of block caving mines, some large seismic events are located in the cave back during the early stages of its progression. Obviously similar events cannot be repeated after the cave is established. Therefore, these events need to be excluded from the observed catalogue that is used to evaluate seismic hazard for the stages of mature cave. 


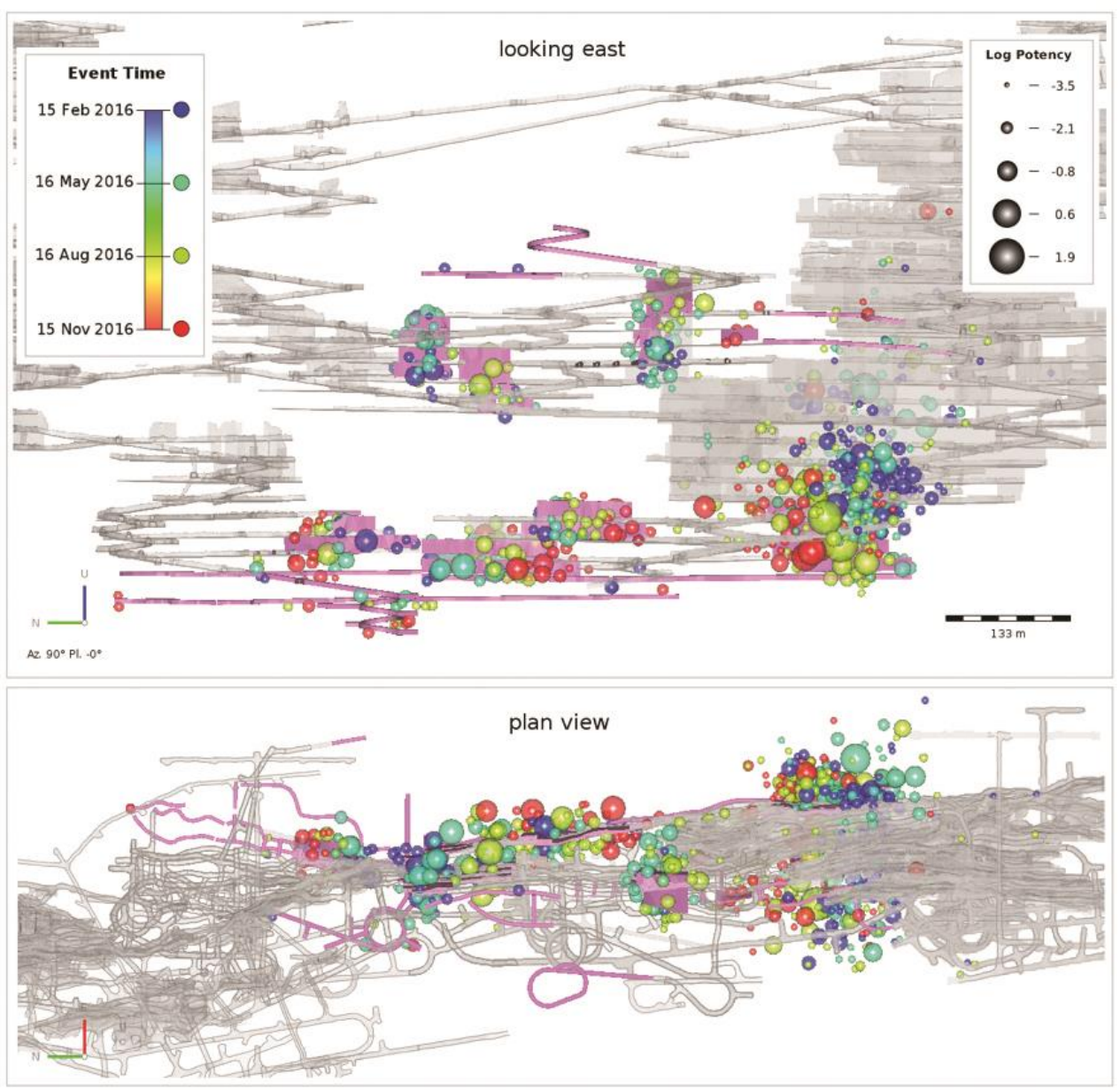

Figure 3 Results of modelling of expected seismicity for quarterly mining steps in 2016. Events are sized according to $\log P$ and coloured according to time. The existing and future mining are displayed by grey and pink wireframes respectively

- The modelling spatial domain is uniformly discretised with step $\Delta X$. The considered example has $\Delta X=20 \mathrm{~m}$, which resulted in 47,470 grid points, each corresponding to $8,000 \mathrm{~m}^{3}$ volume.

- For a particular mining step, a time filter is applied to the combined catalogue starting from the beginning of the catalogue (1 July 2011 in the considered case) to the time of the mining step (e.g. for the $1^{\text {st }}$ mining step the time interval is from 1 July 2011 to 1 April 2016, for the $2^{\text {nd }}$ mining step the interval is from 1 July 2011 to 1 July 2016, etc.). The events with potency below the lower cutoff $P_{\min }$ are filtered out. The $P_{\min }$ is evaluated from the potency frequency distribution of observed data.

- For each seismic event the coefficient of influence to every grid point is calculated. The region of influence is a sphere with radius derived from potency $P$ assuming an average inelastic deformation of $100 \mu$ strains $\left(R=\sqrt[3]{3 P /\left(4 \pi * 10^{-4}\right)}\right)$. The influence of an event decays with the radial distance from the source $r$ according to a function $\sqrt{1-r^{2} / R_{\min }^{2}}, 0 \leq r \leq R_{\min }, R_{\text {min }}=$ $\max (R, 0.866 \Delta X)$. The coefficient 0.866 ensures that each event contributes to at least one grid node. The region of influence can also be chosen according to the source radius derived from the corner frequency of an event (as suggested by Wesseloo 2013), however $R$ derived from potency is expected to be more robust. If the inferred radius $R$ is greater than the grid size $\Delta X$, then it is preferable to take into account the shape of the source. This is done by approximating the source by an ellipsoid with major and intermediate principal axes scaled according to length and width of the slip plane. The slip plane is known for modelled seismic events. In the case of observed data, 
only a few events typically have an estimate of the slip plane available (e.g. from source mechanism inversion or spatial distribution of aftershocks). A spherical region of influence is adopted for all other large observed events. The influence coefficients for a single event are normalised in such a way that their sum over all grid points equals to one.

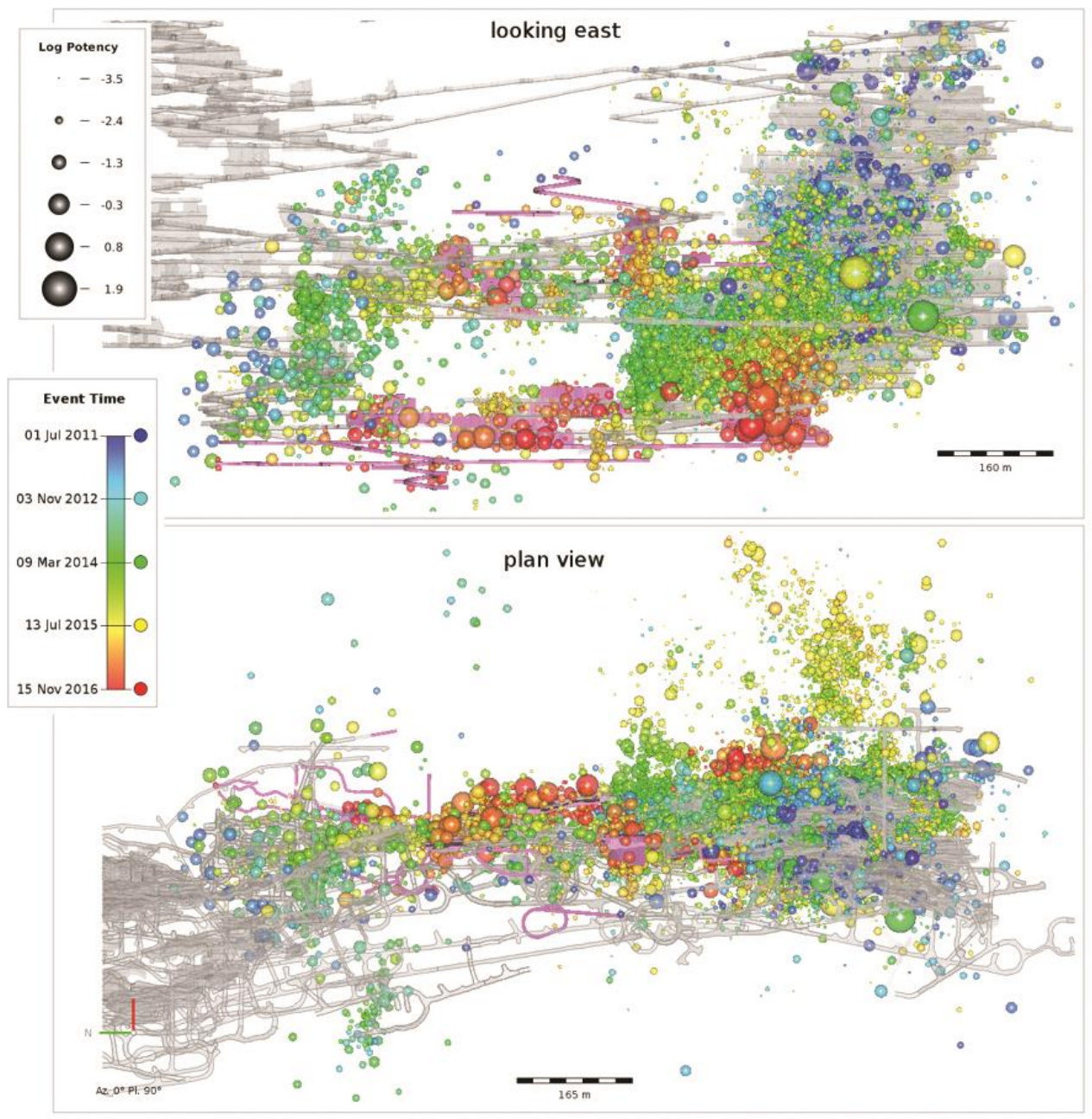

Figure 4 Location, time and sizes of events from the combined catalogue. Events are sized according to $\log P$ and coloured according to time. The existing and planned future mining are displayed by grey and pink wireframes respectively

- The cumulative potency $P_{\Sigma}$ is calculated for each grid point using all seismic events and their influence coefficients. The cumulative potency is normalised to the time interval of interest (exposure time) $T$ resulting in $P_{\sum T}$. In the considered case, the exposure time was set to 92 days.

- It is assumed that the sizes of seismic events within each discretised volume follow an Upper Truncated distribution $N(\geq P)=\alpha\left(P^{-\beta}-P_{\max }^{-\beta}\right)$. The upper potency cutoff $P_{\max }$ and slope $\beta$ are set the same for all grid points. The cutoff $P_{\max }$ is estimated from the combined (observed and modelled) seismic catalogue using the record theory (Section 3.4 of Mendecki 2016). The slope $\beta$ is evaluated from observed data. The parameter $\alpha$ is derived for each grid from the cumulative potency: $\alpha=P_{\sum T}(1 / \beta-1) /\left(P_{\max }^{1-\beta}-P_{\min }^{1-\beta}\right)$, if $\beta \neq 1$ and $\alpha=P_{\sum T} / \ln \left(P_{\max } / P_{\min }\right)$, if $\beta=1$.

- Assuming that the origin time of events can be described by a stationary Poisson process, the probability of occurrence of at least one event with potency equal or greater than $P$ during the exposure time within the grid volume is $\operatorname{Pr}(\geq P, T)=1-\exp \left(-\alpha\left(P^{-\beta}-P_{\max }^{-\beta}\right)\right)$. 
As an example, Figure 5 shows isosurfaces for which the probability of occurrence of at least one event with $\log P \geq 0.1$ in the $8,000 \mathrm{~m}^{3}$ volume in 92 days is 0.001 . The calculations are done for the observed data only (existing mining) and for four planned future quarterly mining steps.

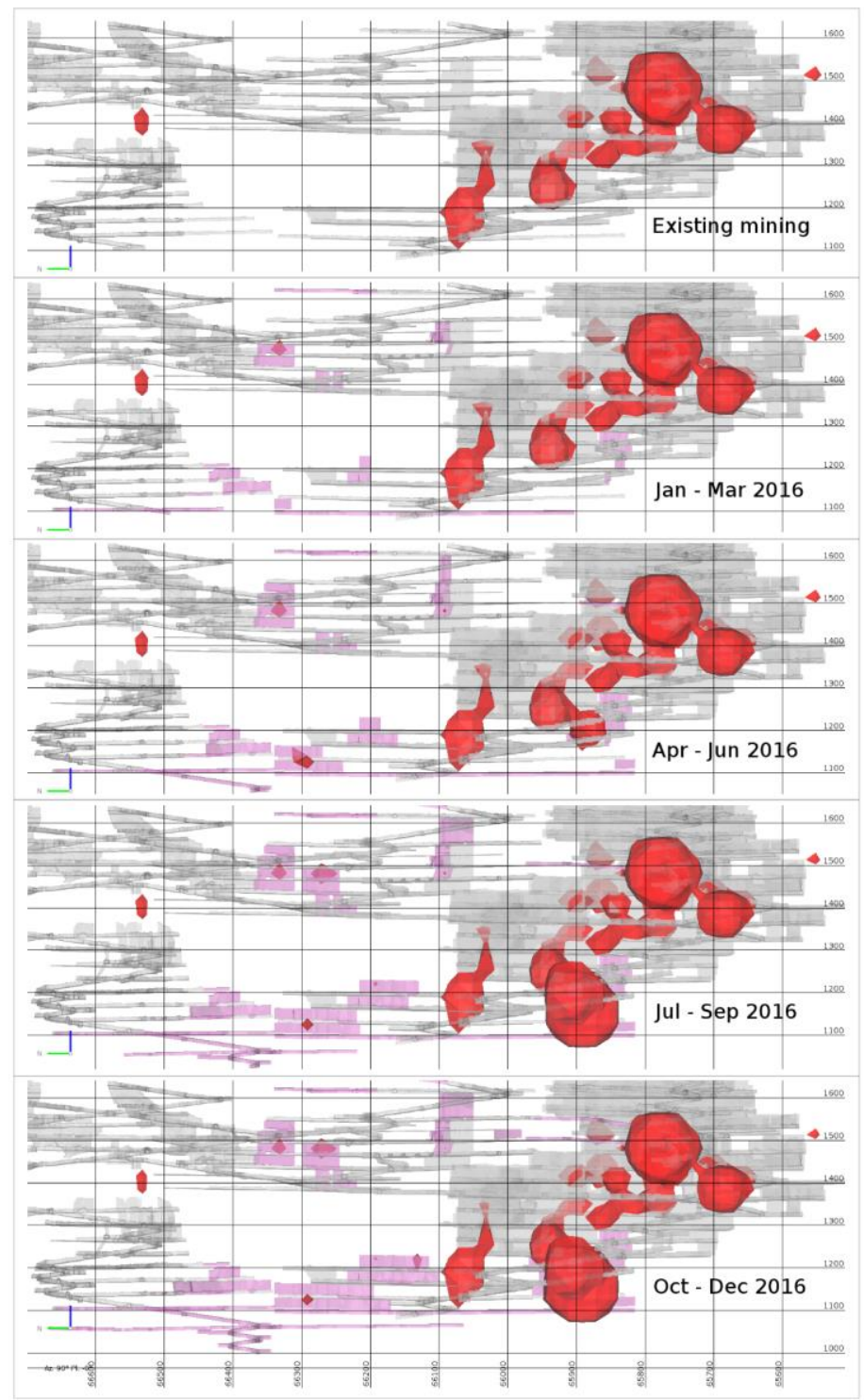

Figure 5 Evolution of seismic hazard for the planned mining sequence. The red wireframes represent isosurfaces for which the probability of occurrence of at least one event with $\log P \geq 0.1$ within $8,000 \mathrm{~m}^{3}$ in 92 days is 0.001 . The existing and planned future mining are displayed by grey and pink wireframes respectively

One may consider the calculated probabilities as being too low. However, it needs to be kept in mind that they correspond to the unit grid volume $\left(8,000 \mathrm{~m}^{3}\right.$ in the considered case). The calculated probabilities can be converted to the probabilities of occurrence of at least one event within the larger areas of interest (e.g. region around decline or specific production area) and subsequently compared with the categories of likelihood of the risk assessment matrix adopted at the mine.

\subsection{Quantification of ground motion hazard}

The seismic hazard can be translated to ground motion hazard. Such a translation requires a relation between the intensity of ground motion and parameters of a seismic source. In our case it is preferable to 
use the Ground Motion Prediction Equation (GMPE) that is based on seismic potency and includes the term constraining the near-source ground motion (Section 6.7 of Mendecki 2016):

$$
\log P G V=c_{P} \log P-c_{R} \log \left[R+c_{l} P^{\frac{1}{3}}\right]+c,
$$

where:

$P G V$ is the peak ground velocity.

$P$ is the seismic potency.

$R$ is the hypocentral distance.

The GMPE needs to be calibrated for the mine, providing not only the optimal coefficients $c_{P}, c_{R}, c_{l}$ and $c$, but also their standard deviations.

A naive way to assess ground motion hazard is to calculate PGV for the modelled events shown in Figure 3. However, this approach does not take into account the contribution of historical seismicity to hazard. Also the catalogue of modelled events does not reflect possible variation of the expected seismicity due to the variation of modelling parameters. A more appropriate approach would be to consider possible realisations of seismicity based on the available models of seismic hazard (e.g. the ones shown in Figure 5). For illustration purposes, Figure 6 shows four realisations of seismicity corresponding to seismic hazard for the October to December 2016 mining step (bottom of Figure 5).

The Monte Carlo simulation was utilised to assess ground motion hazard. This is based on the repetition of the following steps:

1. The possible realisation of seismicity is derived from the model of seismic hazard for a given mining step.

2. The parameters of GMPE (coefficients $c_{P}, c_{R}, c_{l}$ and $c$ ) are disturbed according to their standard deviations.

3. The PGV is calculated using the disturbed GMPE at each grid point for each event with $P \geq P_{d m g}$ from the derived realisation of seismicity. The $P_{d m g}$ limits the size of the smallest potentially damaging event. In the considered example we use $\log P_{d m g}=-0.5$.

4. The maximum PGV is saved for each grid point.

Steps one to four are repeated $N$ times, which resulted in distribution of $N$ PGV values for each grid point. These are converted to the probabilities of exceedance of particular levels of PGV.

As an example the results of calculations for the 0.025 probability of exceedance of PGV in 92 days for the demonstration case are shown in Figure 7. In this case the Monte Carlo simulation included 2,000 runs.

The probability of 0.025 used in the demonstrated case corresponds to the bottom of the 'unlikely' category of likelihood of the risk assessment matrix used at Renison. The calculations of exceedance of PGV can also be done for other categories of likelihood, e.g. 'likely', 'possible', 'rare', which allows a geotechnical engineer to assess the risk of potentially damaging ground motion associated with seismic events. 

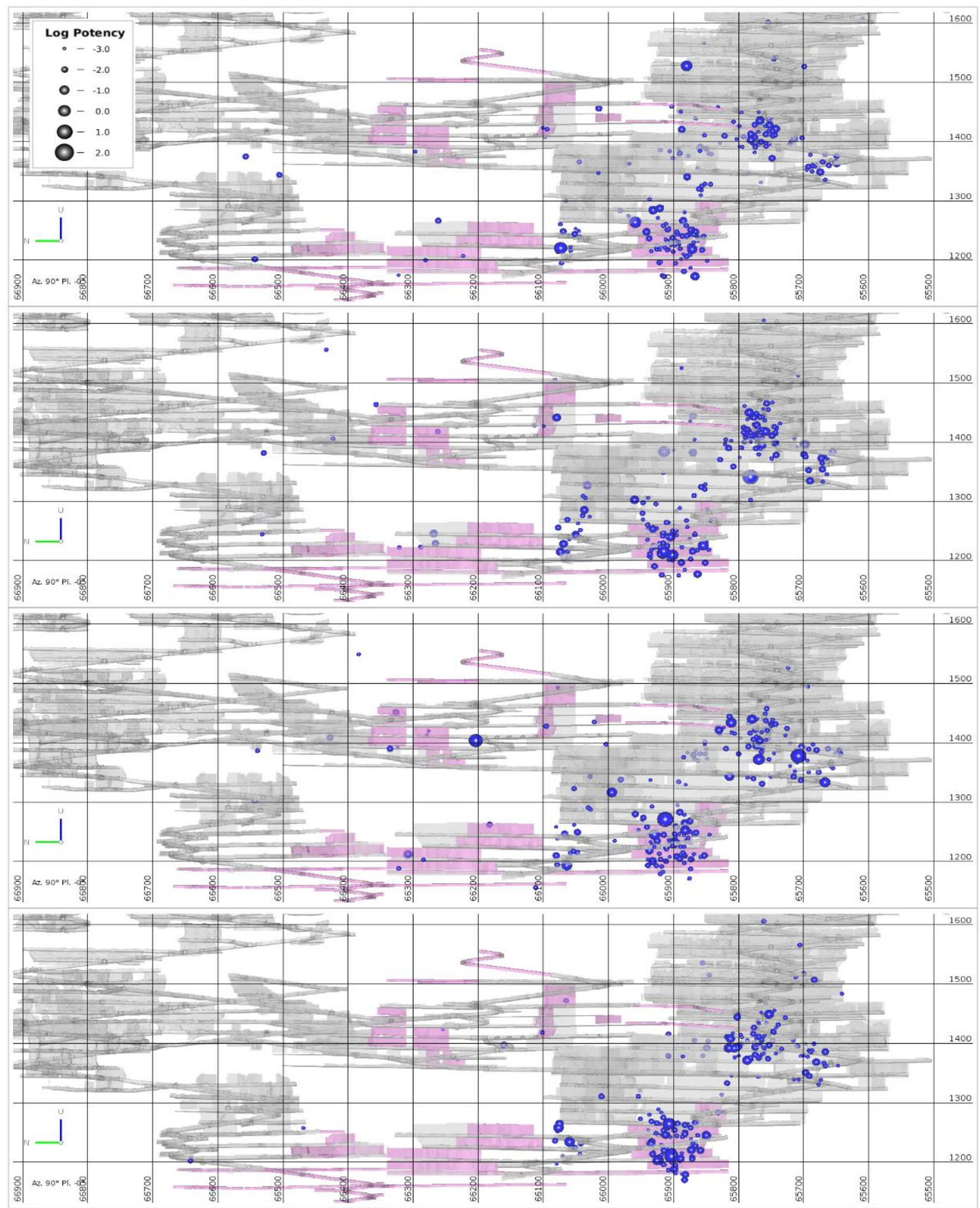

Figure 6 Examples of realisations of seismicity corresponding to the seismic hazard for the October to December 2016 mining step. Only four realisations are shown although the assessment of ground motion hazard includes several thousand simulations. Each realisation consists of the 200 largest events 


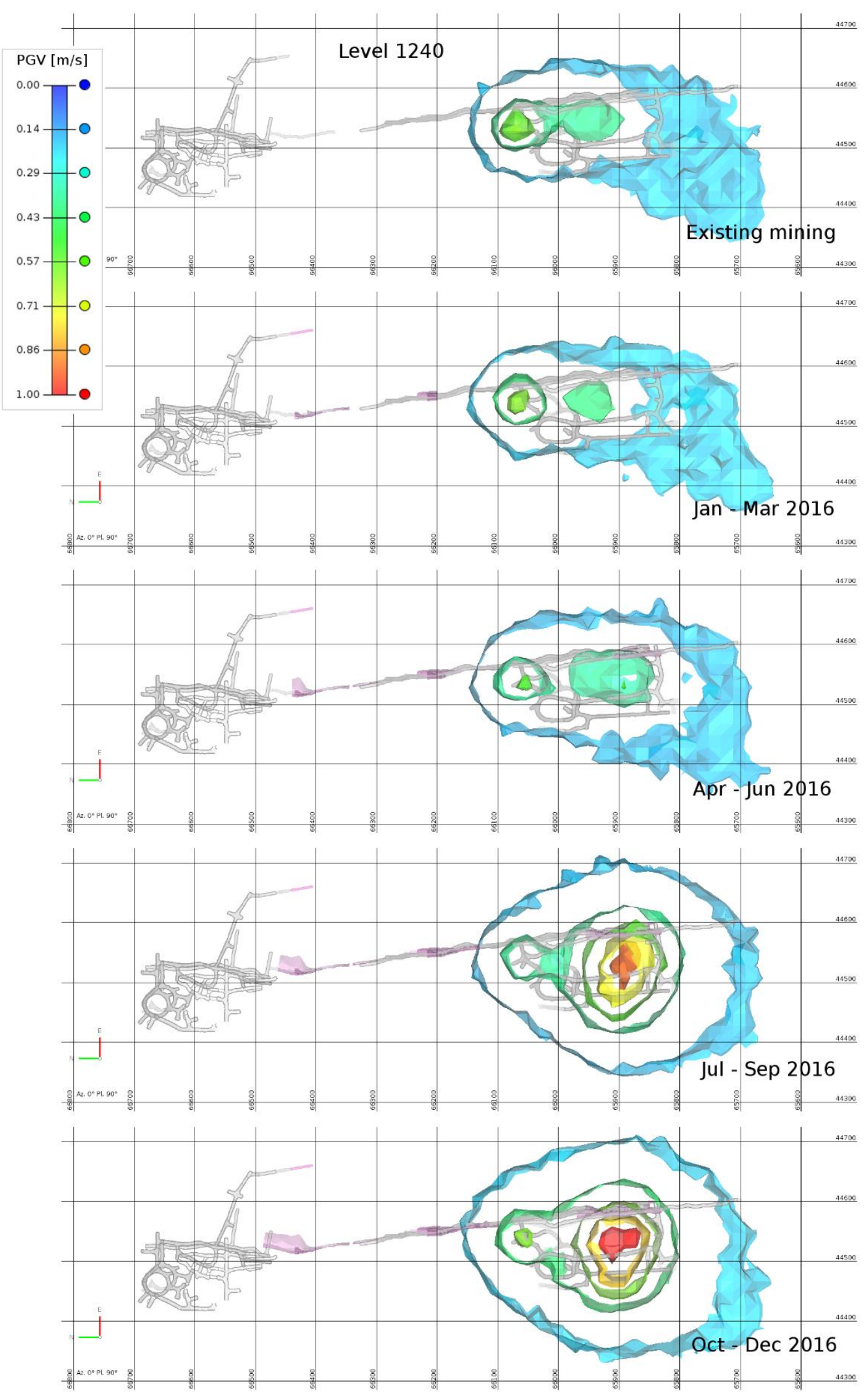

Figure 7 Evolution of ground motion hazard for the planned quarterly mining steps. The wireframes represent isosurfaces of PGV (in $\mathrm{m} / \mathrm{s}$ ) for which the probability of exceedance in 92 days is 0.025 (which corresponds to the bottom of the 'unlikely' category from the risk assessment matrix adopted at the mine). The plots are done for Level 1,240 of the mine. The existing and planned future mining are displayed by grey and pink wireframes respectively 


\section{$3 \quad$ Testing of hazard forecasts}

The ability to use the risk assessment matrix is the major, but not the only benefit of following the exact definition of hazard and delivering results of hazard assessment not in qualitative terms (e.g. blue and red areas), but in terms of probabilities. The other benefit is that the evaluated probabilities of occurrence of seismic events or exceedance of ground motion can be rigorously tested after the period of forecast is expired and actual seismic response to planned mining is recorded. This allows for the comparison of different forecasts (e.g. based on different input parameters of modelling of seismicity) and the monitoring of their performance.

The testing of forecasts of seismic hazard has received significant attention in the last 10 years in crustal seismology. The procedures of testing of the earthquake forecasts were established and applied to a set of forecasts issued prospectively (in advance) by various groups of researchers for California in terms of Regional Earthquake Likelihood Models (RELM) project (Schorlemmer et al. 2007; Field 2007; Schorlemmer et al. 2010). Later, the principles and methods of prospective testing of seismic hazard were transformed into a routine mode under the Collaboratory for the Study of Earthquake Predictability (CSEP) initiative (Zechar et al. 2010). Currently, it runs in a number of regions of the world.

There are several tests established in the CSEP that allow quantification of the performance of particular forecast. Often they are applied in a comparative mode, where one forecast is compared with the reference one. As the reference forecast, it is not recommended using an unskilled forecast (e.g. uniform spatial distribution of earthquakes), but rather a forecast derived from the smoothed observed seismicity (assumption that 'tomorrow will be like today'). One of the CSEP tests - Area Skill Score (Zechar \& Jordan 2008; Zechar 2010) - has been applied to the retrospective forecasts of seismic hazard for Renison mine for 2014 and 2015. The seventeen events with $\log P \geq 0.0$ (11 recorded in 2014 and six in 2015) were used as targets. The locations of these events in relation to the mining areas are shown in Figure 8.
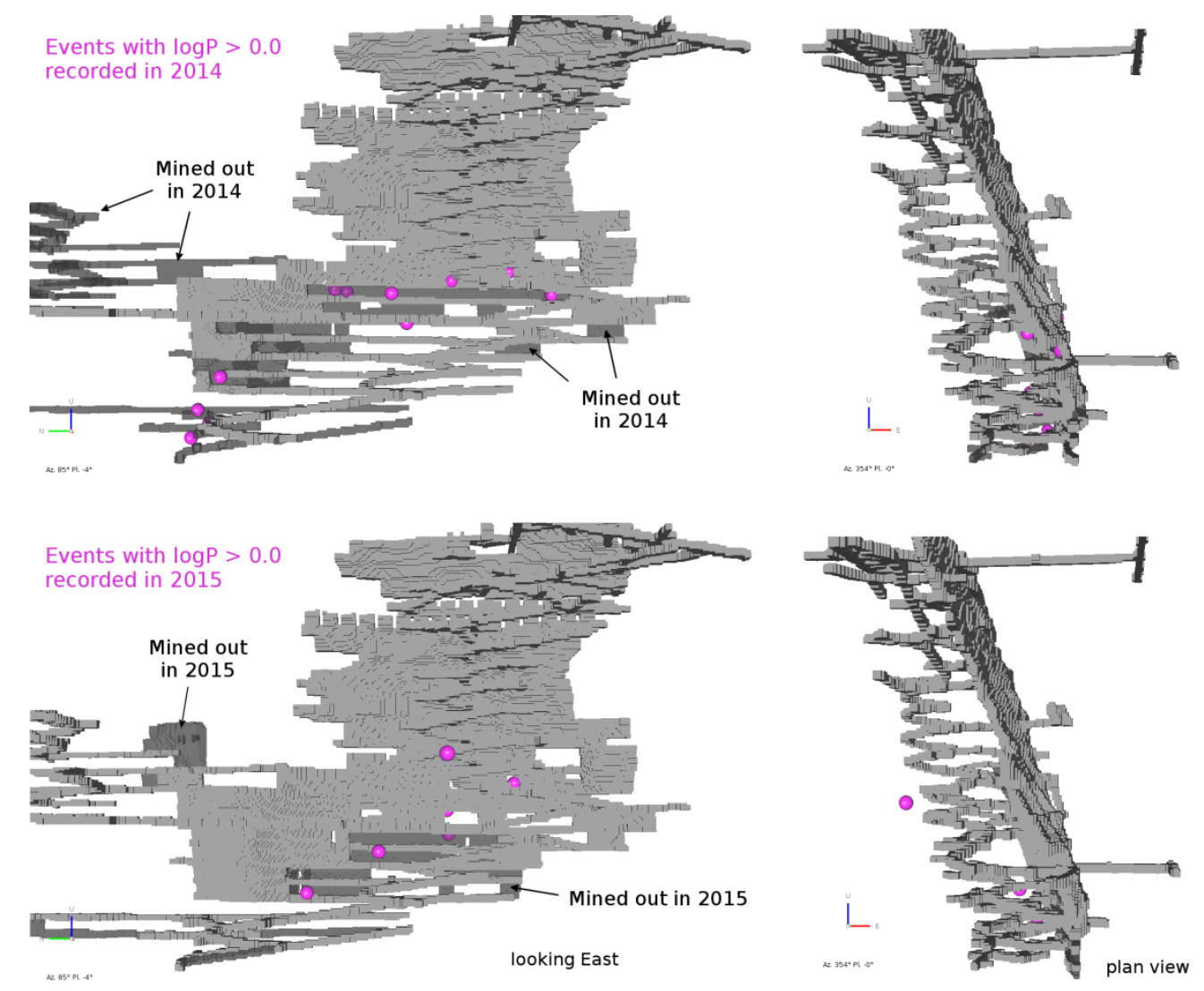

Figure 8 Location of events used as targets for evaluating forecast performance. The excavations that existed before 2014 (top) and before 2015 (bottom) are shown as grey wireframes. The excavations mined out in 2014 (top) and in 2015 (bottom) have darker colour 
The results of testing are presented in Figures 9 and 10.
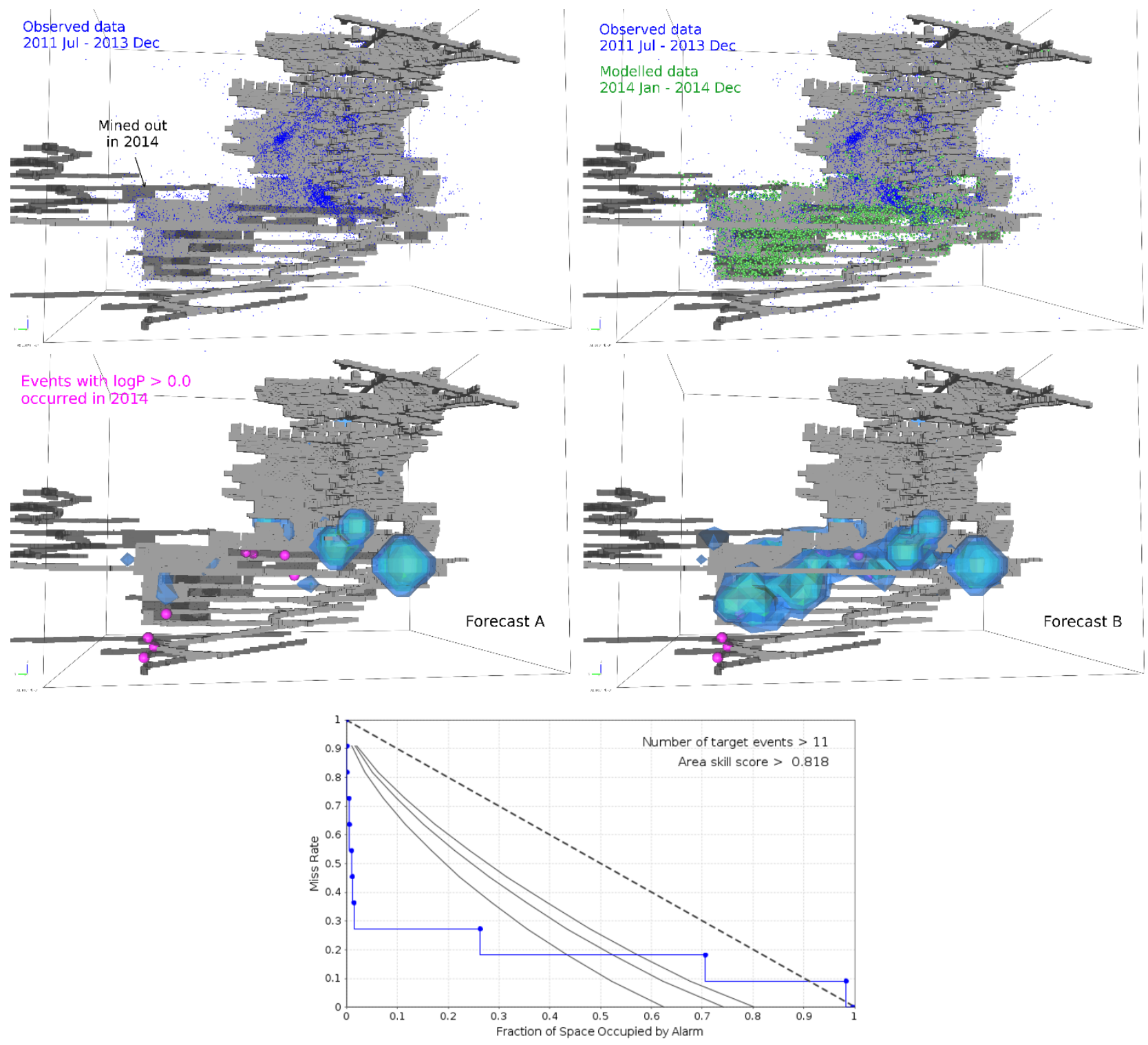

Figure 9 Testing of the forecast of seismic hazard for 2014. The excavations that existed before 2014 are shown as grey wireframes. The excavations mined out in 2014 have darker colour. Top left and right plots show seismicity recorded before 2014 as blue dots. The green spheres in the top right plot represent seismic events modelled using the Salamon-Linkov method for the actual mining done in 2014. The middle plots show the results of transformation of the observed seismicity (left) and combined (observed and modelled) seismicity (right) to seismic hazard using the approach described in the previous section. The isosurfaces for which the probabilities of occurrence of at least one event with $\log P \geq 0.0$ within $8,000 \mathrm{~m}^{3}$ in 92 days equal to $0.001,0.0033,0.0067$ and 0.01 are visualised as coloured wireframes. The pink spheres show actual events with $\log P \geq \mathbf{0 . 0}$ that occurred in 2014. These events were used in the Molchan diagram in the bottom. The diagonal dashed line in the diagram represents an unskilled forecast and the grey solid lines correspond to its 90,95 , and $99 \%$ confidence bounds

Figure 9 evaluates the performance of the forecast of seismic hazard for 2014 derived from the combination of seismic data observed before 2014 and modelled data for 2014 (forecast B) relative to the reference forecast derived from only the data observed before 2014 (forecast $A$ ). There were 11 seismic events with $\log P \geq 0.0$ recorded in 2014 (pink spheres). These tend to locate in the areas where forecast $\mathrm{B}$ prescribes high probability compared to forecast $A$. The Molchan diagram presented in the bottom of the 
figure quantifies this difference. It shows that eight out of 11 events occurred in the areas where the probability of occurrence of events according to forecast $B$ is significantly higher than the probability according to the reference forecast $A$. A single event occurred in the place where forecast $A$ has higher probability than forecast $B$. The overall score of the performance (Area Skill Score) is 0.826 , which indicates that forecast $B$ provides significant gain relative to forecast $A$.

A similar performance is obtained for the retrospective forecast done for 2015 (Figure 10), when six events with $\log P \geq 0.0$ were recorded. Five of these are located in the areas where the probability of occurrence according to forecast B (observed and modelled data) is significantly higher than for forecast A (observed data only).

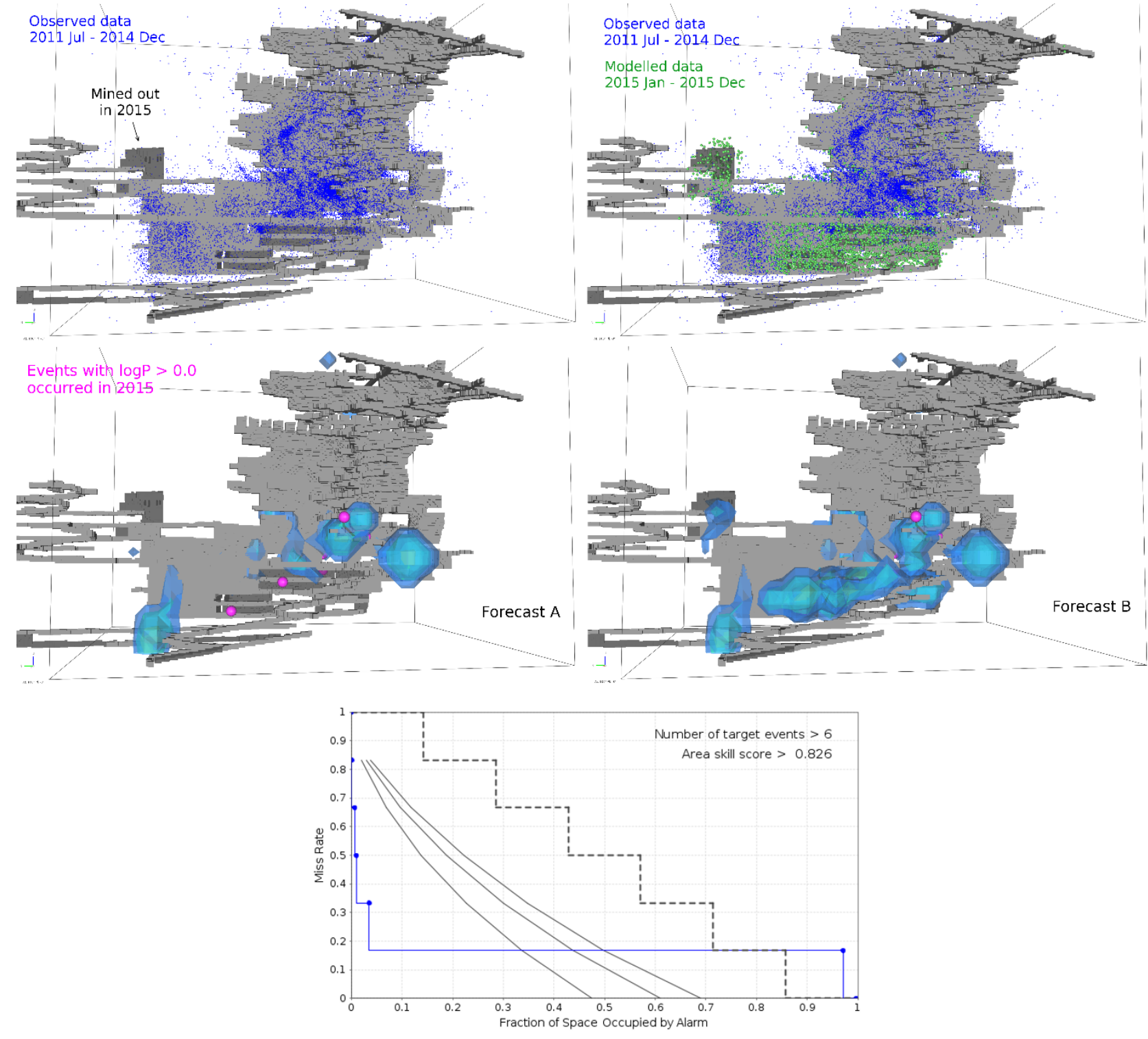

Figure 10 Results of testing of the forecast of seismic hazard for 2015. All notations and conventions are the same as in Figure 9 


\section{$4 \quad$ Discussion and concluding remarks}

There are several assumptions in the suggested approach of seismic hazard assessment that are worth discussing.

1. The current version of the Salamon-Linkov method presumes that the failure in rock mass can be described by Displacement Discontinuity elements with tangential slip component. Thus, all modelled seismic and aseismic events have pure double-couple source mechanisms. It is well known that episodes of sudden inelastic deformation in mines quite often have a significant volumetric component (e.g. pillar or abutment failure). The resulting seismic events demonstrate substantial isotropic implosive components and they are described by a closing crack point source model rather than a double-couple model. The assumption of pure slip sources can be important for the interpretation of large events or seismicity, when the characteristics of modelled and observed events are compared. In regards to the assessment of seismic hazard such an assumption should not be critical. It is expected that the total seismic potency of volumetric failure should not depend much on how it is modelled (either explicitly or as a combination of slips along planes). Thus, the derived hazard should also not be significantly affected by the details of the modelling of volumetric failure sources. The testing of this assumption is within the scope of future work.

2. A constant slope $(\beta)$ of the potency frequency distribution is assumed for all parts of the hazard assessment domain (Section 2.2). This helps to ensure that the calculations are robust. The spatial variability of $\beta$ can be incorporated in the hazard assessment method, however it will require a careful treatment of the uncertainty of $\beta$. As it is shown in Section 2.4.1 of Mendecki (2016), over 1,000 events may be needed to get the $95 \%$ confidence errors in $\beta$ smaller than 0.1 . Thus, the robust assessment of $\beta$ for each grid point will require a spatial smoothing of the data, i.e. involvement of events that may be located far from the analysed grid point. It is useful to note that there is no consensus in global seismology with regards to the spatial variability of the slope of magnitude frequency distribution ( $b$-value). Some researchers consider the spatial inhomogeneity of $b$-values as an established fact and adopt it in their forecasts of seismic hazard (Wiemer \& Schorlemmer 2007). The others think that the 'null hypothesis' of universal uniform slope $\beta$ has not been rejected and the apparent variability of $\beta$ may originate from small datasets or mixed earthquakes populations (Bird \& Kagan 2004). Therefore, they prefer to use a fixed slope for the assessment of seismic hazard (Kagan et al. 2007).

3. In general, the supposition that the occurrence of events in time can be described by a stationary Poisson process disagrees with the observations. The rate of seismic events in mines usually changes after large blasts or large seismic events. Nevertheless, there are still two rationales behind the adopted assumptions of constant rate and independence of times of seismic events. Firstly, the major contributors to the estimate of hazard are the larger events and they do tend to agree with a stationary Poisson process (Mendecki \& Lötter 2011) in contrast to the smaller events. Secondly, a stationary Poisson process represents a simple model which makes the calculations of hazard more robust. It is possible to design more complex models which will require extra input settings (e.g. non-stationary Poisson processes). The testing of the hazard forecasts of such models against the current approach will allow one to assess their utility. This is within the scope of future work.

The suggested method of hazard assessment also has multiple input parameters and settings. The following of which are expected to have significant effect on the results:

- Location, source parameters and mechanisms of the largest recorded seismic events. The cumulative seismic potency $P_{\Sigma}$ observed at mines is quite often dominated by the few largest events. According to the suggested approach these will have major contributions to the hazard. Therefore, careful evaluation of seismic potency (for instance using data from remote sites) is 
important. The reconstruction of spatial distribution of the largest sources (e.g. identification of orientation and dimensions of slip plane) is also critical for the hazard estimates.

- The Ground Motion Prediction Equation, especially its terms defining the ground motion in the near-source region. Slip inversion of the sources of large events using near-source waveforms can help to constrain these terms.

The assessment of seismic hazard is sometimes reduced to a qualitative separation of areas of the mine with higher and lower hazard in space, or periods of higher and lower hazard in time. This paper emphasises the importance of complying with the definition of hazard, i.e. delivering the probabilities of occurrence of potentially damaging seismic events or ground motion. First of all, the calculated probabilities can be matched with the likelihoods of hazard specified in the risk assessment matrix established at the mine. This can guide a geotechnical engineer in the required actions. Secondly, the quantitative hazard assessment allows a testing of its performance, i.e. to objectively conclude which of the alternative forecasts is better and can be used for mine planning purposes. The testing described in this paper may also help to optimise the hazard assessment procedure. If the testing of two methods of hazard assessment - simple/cheap and complex/expensive - suggests that their skills are similar (e.g. the Molchan diagram shows that there is no gain of one forecast relative to the other), then it makes sense to adopt the simple/cheap method for routine application.

The mines utilising the assessment of seismic hazard should expect that the forecast will fail one day. There were recent cases in Australian and South African mines, when very large seismic events were associated with slip along major geological structures that were not previously known and were not illuminated by seismicity in advance. In such cases any hazard assessment approach (based only on seismic data, or on the combination of observed and modelled data, or only on modelled data) will underestimate the hazard. The only constructive solution is to accept the possibility of failure of the forecasts and adopt the strategy of continuous monitoring and improvement of the forecasts performance (i.e. implement continuous cycle 'forecast for future interval $A \rightarrow$ test of the forecast in the end of $A \rightarrow$ correct the model $\rightarrow$ forecast for future interval $B \rightarrow$ test of the forecast in the end of $B \rightarrow \ldots$ ').

\section{Acknowledgement}

The presented work has been done under IMS Patronage project 'Modelling and Assimilation of Seismicity using Boundary Element Method (A-MAS)' supported by El Teniente Mine (Chile), LKAB (Sweden) and Newcrest Mining (Australia).

Renison mine kindly permitted the use of the data. I am grateful to the senior geotechnical engineer of the mine, Kevin Stacey, for providing the required information and for interesting discussion of the results.

Aleksander Mendecki provided numerous fruitful suggestions regarding the evaluation of seismic and ground motion hazard. Ernest Lötter and Gys Basson helped with the implementation of the calculation and visualisation of seismic and ground motion hazard. Tjaart de Wit improved the use of English in the manuscript.

The constructive comments and suggestions from an anonymous reviewer were highly appreciated.

\section{References}

Arndt, S, Louchnikov, V, Weller, S \& O'Hare, A 2013, 'Forecasting mining induced seismicity from modelled energy release in high stress stope extraction', in A Malovichko \& D Malovichko (eds), Proceedings of the Eighth International Symposium on Rockbursts and Seismicity in Mines, Geophysical Survey of Russian Academy of Sciences, Obninsk, and Mining Institute of the Ural Branch of the Russian Academy of Sciences, Perm, pp. 267-272.

Beck, DA \& Brady, BHG 2002, 'Evaluation and application of controlling parameters for seismic events in hard-rock mines', International Journal of Rock Mechanics and Mining Sciences and Geomechanics Abstracts, vol. 39, no. 5, pp. 633-642.

Beck, D, Reusch, F \& Arndt, S 2007, 'Estimating the probability of mining-induced seismic events using mine-scale, inelastic numerical models', in Y Potvin (ed.), Proceedings of the Fourth International Seminar on Deep and High Stress Mining, Australian Centre for Geomechanics, Perth, pp. 31-42. 
Bird, P \& Kagan, YY 2004, 'Plate-tectonic analysis of shallow seismicity: Apparent boundary width, beta, corner magnitude, coupled lithosphere thickness, and coupling in seven tectonic settings', Bulletin of the Seismological Society of America, vol. 94, no. 6, pp. 2380-2399.

Board, MP 1994, Numerical examination of mining-induced seismicity, PhD thesis, University of Minnesota, Minneapolis, pp. 480.

Field, EH 2007, 'Overview of the working group for the development of regional earthquake likelihood models (RELM)', Seismological Research Letters, vol. 78, no. 1, pp. 7-16.

Kagan, YY, Jackson, DD \& Rong, Y 2007, 'A testable five-year forecast of moderate and large earthquakes in Southern California based on smoothed seismicity', Seismological Research Letters, vol. 78, no. 1, pp. 94-98.

Levkovitch, V, Beck, D \& Reusch, F 2013, 'Numerical simulation of the released energy in strain-softening rock materials and its application in estimating seismic hazards in mines', in A Malovichko \& D Malovichko (eds), Proceedings of the Eighth International Symposium on Rockbursts and Seismicity in Mines, Geophysical Survey of Russian Academy of Sciences, Obninsk, and Mining Institute of the Ural Branch of the Russian Academy of Sciences, Perm, pp. 259-266.

Linkov, AM 2005, 'Numerical modeling of seismic and aseismic events in geomechanics', Journal of Mining Sciences, vol. 41, no. 1, pp. 14-26.

Linkov, AM 2013, 'Keynote lecture: Numerical modelling of seismicity: Theory and applications', in A Malovichko \& D Malovichko (eds), Proceedings of the Eighth International Symposium on Rockbursts and Seismicity in Mines, Geophysical Survey of Russian Academy of Sciences, Obninsk, and Mining Institute of the Ural Branch of the Russian Academy of Sciences, Perm, pp. 197-218.

Linkov, AM, Rybarska-Rusinek, L \& Zoubkov, VV 2016, 'Reasonable sets of input parameters and output distributions for simulation of seismicity', International Journal of Rock Mechanics and Mining Sciences, vol. 84, pp. 87-94.

Malovichko, D \& Basson, G 2014, 'Simulation of mining induced seismicity using Salamon-Linkov method', in M Hudyma \& Y Potvin (eds), Proceedings of the Seventh International Conference on Deep and High Stress Mining, Australian Centre for Geomechanics, Perth, pp. 667-680.

Mendecki, AJ 2016, Mine Seismology Reference Book: Seismic Hazard, Institute of Mine Seismology, pp. 88.

Mendecki, AJ \& Lötter, EC 2011, 'Modelling seismic hazard for mines', Proceedings of the Australian Earthquake Engineering Society 2011 Conference, 18-20 November 2011, Barossa Valley, viewed 6 November 2016, http://www.aees.org.au/ wp-content/uploads/2013/11/38-MENDECKI-LOTTER.pdf

Salamon, MDG 1993, 'Keynote address: Some applications of geomechanical modelling in rockburst and related research', in P Young (ed.), Proceedings of the Third International Symposium on Rockbursts and Seismicity in Mines, Balkema, Rotterdam, pp. 297-309.

Schorlemmer, D, Gerstenberger, MC, Wiemer, S, Jackson, DD \& Rhoades, DA 2007, 'Earthquake likelihood model testing', Seismological Research Letters, vol. 78, no. 1, pp. 17-29.

Schorlemmer, D, Zechar, JD, Werner, MJ, Jackson, DD, Field, EH, Jordan, TH \& the RELM Working Group 2010, 'First results of the Regional Earthquake Likelihood Models experiment', Pure and Applied Geophysics, vol. 167, no. 8/9, pp. 859-876.

Sellers, EJ \& Napier, JAL 2001, 'A point kernel representation of large-scale seismic activity in mining', in G van Aswegen, RJ Durrheim \& WD Ortlepp (eds), Proceedings of the Fifth International Symposium on Rockbursts and Seismicity in Mines, Southern African Institute of Mining and Metallurgy, Johannesburg, pp. 405-411.

Spottiswoode, S 2001, 'Keynote address: Synthetic seismicity mimics observed seismicity in deep tabular mines', in G van Aswegen, RJ Durrheim \& WD Ortlepp (eds), Proceedings of the Fifth International Symposium on Rockbursts and Seismicity in Mines, Southern African Institute of Mining and Metallurgy, Johannesburg, pp. 371-377.

Spottiswoode, SM \& Drummond, M 2014, 'Pillar behaviour and seismicity in platinum mines', Journal of the Southern African Institute of Mining and Metallurgy, vol. 14, pp. 801-809.

Wesseloo, J 2013, 'Towards real-time probabilistic hazard assessment of the current hazard state for mines', in A Malovichko \& D Malovichko (eds), Proceedings of the Eighth International Symposium on Rockbursts and Seismicity in Mines, Geophysical Survey of Russian Academy of Sciences, Obninsk, and Mining Institute of the Ural Branch of the Russian Academy of Sciences, Perm, pp. 307-312.

Wiemer, S \& Schorlemmer, D 2007, 'ALM: An asperity-based likelihood model for California', Seismological Research Letters, vol. 78, no. 1, pp. 134-140.

Zechar, JD, 2010, 'Evaluating earthquake predictions and earthquake forecasts: a guide for students and new researchers', Community Online Resource for Statistical Seismicity Analysis, viewed 6 November 2016, http://www.corssa.org/ articles/theme_6

Zechar, JD \& Jordan, TH 2008, 'Testing alarm-based earthquake predictions', Geophysical Journal International, vol. 172, pp. 715-724.

Zechar, JD, Schorlemmer, D, Liukis, M, Yu, J, Euchner, F, Maechling, PJ \& Jordan, TH 2010, 'The Collaboratory for the Study of Earthquake Predictability perspective on computational earthquake science', Concurrency and Computation: Practice and Experience, vol. 22, pp. 1836-1847. 
